\title{
Effect of Four Commonly Used Dissolution Media Surfactants on Pancreatin Proteolytic Activity
}

\author{
Maya Guncheva ${ }^{1,2,3}$ and Erika Stippler ${ }^{2}$
}

Received 9 June 2016; accepted 14 August 2016; published online 1 September 2016

\begin{abstract}
Proteolytic enzymes are often used in dissolution testing of cross-linked gelatin capsules that do not conform to the dissolution specification. Their catalytic activity, however, can be affected when they are added to a dissolution media containing solubility enhancers, such as surfactants. The aim of this study was to assess the activity of pancreatic proteases in presence of four commonly used surfactants. We found that pancreatin exhibits remarkable proteolytic activity in the presence of Tween 80, even at the concentrations as high as 250 times its critical micelle concentration $(\mathrm{cmc})$ in water, whereas, Triton X-100 enhanced the proteolytic activity of pancreatin when added at concentrations above its $\mathrm{cmc}$ in water. Both surfactants are non-ionic surfactants. On the other hand, sodium dodecyl sulfate (SDS) and cetyltrimethylammonium bromide (CTAB), which are ionic surfactants, have a detrimental effect on the proteolytic activity of pancreatin. For example, a $50 \%$ reduction of the pancreatin activity was found in samples which contain a minor amount of SDS $(0.05 \% \mathrm{w} / \mathrm{v})$ in comparison to a surfactant-free reaction. Additionally, no activity was observed for the pancreatin-SDS samples which were incubated for $30 \mathrm{~min}$ at $40^{\circ} \mathrm{C}$ prior to testing. CTAB had an impact on pancreatin activity at concentrations higher than its cmc. Data from this manuscript can be used as a benchmark for optimization of the dissolution procedures that require use of both surfactants and enzymes.
\end{abstract}

KEY WORDS: dissolution testing; gelatin capsules; pancreatin; surfactants.

\section{INTRODUCTION}

Gelatin is one of the commonly used materials for preparation of some solid oral dosage forms, such as hardshell and soft-shell gelatin capsule formulations (1-3). Amongst the main advantages of using gelatin for the capsule shell manufacturing are its biocompatible properties, its biodegradable nature, and its lack of toxicity (4). Also, the side chains of amino acids that comprise the gelatin molecules offer many functional groups that can be subjected to modification, to obtain gelatin-co-polymer formulations for time-controlled drug release or ligand-functionalized gelatin nanocarriers for targeting a specific receptor $(5,6)$.

The main disadvantage of gelatin is that its physicochemical properties can be changed over time, due to side

\footnotetext{
$\overline{{ }^{1} \text { Institute of Organic Chemistry, Centre of Phytochemistry, Bulgarian }}$ Academy of Sciences, Sofia, Bulgaria.

${ }^{2}$ U.S. Pharmacopeial Convention, Rockville, Maryland, USA.

${ }^{3}$ To whom correspondence should be addressed. (e-mail: maiag@orgchm.bas.bg)

ABBREVIATIONS cmc, Critical micellar concentration; CTAB, Cetyltrimethylammonium bromide; pI, Isoelectric point; Triton X-100; Polyethylene glycol tert-octylphenyl ether; Tween 80; Polysorbate 80; USP, United States Pharmacopeia
}

reactions when two or more gelatin molecules become bonded, a phenomenon called cross-linking. Typically, traces of aldehydes catalyze the formation of strong covalent bonds between the side-chain lysyl residues, while trivalent metal ions $\left(\mathrm{Fe}^{+3}\right.$ or $\left.\mathrm{Al}^{+3}\right)$ may form weak chelates with free carboxylic groups of gelatin molecules (7). Pharmaceutically active compounds, excipients, colorants, or packaging materials may be a source of cross-linking reagents for gelatin capsules, or they can be obtained as byproducts of their decomposition during storage conditions (high temperature, humidity, and exposure to UV light) $(7,8)$.

In vitro dissolution testing of highly cross-linked gelatin capsules is usually characterized by a prolonged disintegration time and lower dissolution rate $(9,10)$. However, in vivo testing shows that hard and soft cross-linked capsules are bioequivalent to unstressed capsules, i.e., no differences in bioavailability of the tested drugs between fresh and crosslinked gelatin capsules are observed (11). To overcome this limitation and artefact of the in vitro test, proteolytic enzymes are generally added to the dissolution medium. They are able to catalyze the hydrolysis of the peptide bonds in large peptide substrates such as gelatin and therefore to facilitate the drug release from cross-linked gelatin capsules.

According to Dissolution <711> (12) and Disintegration and Dissolution of Dietary Supplements <2040> (13) in the 
United States Pharmacopeia (USP), dissolution experiments of cross-linked gelatin capsules are applied to an in vitro model that involves the addition of an enzyme to the dissolution medium. In general the recommendation is to use pepsin in a concentration of not more than 750,000 units/ $\mathrm{L}$ (using a hemoglobin substrate) for media having acidic $\mathrm{pH}$ value and pancreatin in a concentration of not more than 1750 USP units/L (using casein substrate) in buffer media with $\mathrm{pH}$ values of 6.8 or higher.

Dissolution testing of some drug formulations, specifically of those that have an active ingredient that is hydrophobic or the drug substance has a limited aqueous solubility, requires the addition of surfactants to the dissolution medium (14). The surfactant being used needs to be selected carefully $(9,15,16)$. In situations when both surfactants and enzymes are required for the dissolution test, a two-step testing method may have to be considered to take advantage of the individual characteristics of the enzyme and the surfactant.

Previously, it has been demonstrated that polysorbate 80 (Tween 80), polyethylene glycol tert-octylphenyl ether (Triton X-100), and cetyltrimethylammonium bromide (CTAB) preserve or slightly increased the activity of pepsin, depending on the amount of surfactant added to the simulated gastric fluid media tested (17). At the same time, sodium dodecyl sulfate (SDS) strongly inhibited the activity of pepsin, even at concentration lower than the SDS critical micellar concentration (cmc) (17).

To this date, there is no systematic data on the activity of pancreatin in the literature in presence of surfactants typically used for dissolution testing. Pancreatin is the term used for the complex of digestive enzymes secreted by two types of pancreatic cells and contains hydrolytic enzymes with various substrate specificities, such as proteases (trypsin, chymotrypsin, elastin, carboxypeptidase), cholesterol esterase and lipases (pancreatic lipase and phospholipase), pancreatic amylase, and several nucleases (18). Depending on the source of the pancreatin, the maximum proteolytic activity occurs between $\mathrm{pH} 7$ and 8 (18). The proteases that are present in the solution will simultaneously cleave several of the amide bonds in a gelatin molecule and therefore may accelerate the hydrolysis of the highly cross-linked gelatin.

The aim of this study was to assess the effect of four frequently used surfactants in dissolution testing (Tween 80, Triton X-100, SDS, and CTAB) on the pancreatin proteolytic activity. The proteolytic activity of pancreatin was evaluated for four different pancreatin-surfactant ratios after immediate mixing of the surfactant with the pancreatin as well as after a 30-min incubation (at $40^{\circ} \mathrm{C}$ ) of the pancreatin-surfactant mixture. Dissolution tests on typical cross-linked capsule formulation using the combination enzyme-surfactant were out of the scope of this study.

\section{MATERIALS AND METHODS}

\section{Materials}

Pancreatin amylase and protease reference standards, having a protease activity of 358 USP units/mg (Lot. JOJ 108), were obtained from the US Pharmacopeial Convention. Pancreatin from porcine pancreas, with a proteolytic activity of 100 USP units/mg (Sigma, Lot. SLBN4829V), was used as a reagent. One USP unit corresponds to the amount of pancreatin that hydrolyzes an amount of casein per minute under the assay conditions, which gives soluble peptides that have an absorbance at $280 \mathrm{~nm}$ that is equal to the absorbance of $15 \mathrm{nM}$ of tyrosine. Casein, Hammarsten grade (Sigma, Lot. SLBNO832V), was used a substrate. Phosphate buffer solution (50 mM, pH $7.5 \pm 0.02)$ was prepared using monobasic potassium phosphate (Fisher, Lot. 148095) and sodium hydroxide (Fisher, Lot. 158536). Reagent grade 5\% trichloroacetic acid (Aqua Solutions Inc., Lot. 6022637) was used to stop the hydrolysis. CTAB (MP Biomedicals, Lot. MR31911), SDS (Spectrum, Lot. 2DH221), Triton X-100 (Fisher, Lot. 145168), and Tween-80 (Fisher, 147325) were the surfactants tested. Milli Q water was used to prepare all solutions and the buffer. Polytetrafluoroethylene membrane syringe filters (Fisher, Lot. R5KA37845) were used.

\section{Assessment of the Proteolytic Activity of the Pancreatin Reagent}

The stock solutions of the pancreatin reagent, amylase and protease reference standards, and casein substrate were freshly prepared on the date of use in the concentration range recommended in the USP monograph, and the proteolytic activity of pancreatin reagent was evaluated using casein as a substrate (19). Blank samples containing potassium-sodium phosphate buffer (50 mM, pH 7.5), casein substrate solution, and trichloroacetic acid were prepared to calibrate the spectrophotometer. Control samples containing the same amount of substrate and enzyme corresponding to the point of test reactions when TCA was added, prior to the heating step were prepared. The substrate blank solution was used as blank. This was done to take into account the absorbance of the enzymes. The absorbance of each of the samples was measured at $280 \mathrm{~nm}$ using an AGILENT single beam UVvisible spectrophotometer (Model 8453-61103A), in a 1-cm quartz cell.

A calibration curve for the amylase and protease reference standards was constructed by plotting the samples corrected absorbance values at $280 \mathrm{~nm}$ against the corresponding activity, in USP units of proteolytic activity per milliliter, as calculated from the dilution to obtain the final concentration of the enzyme preparation in the reaction mixture. Using this standard curve, the proteolytic activity of the pancreatin reagent sample was estimated, taking into account the dilution factor. All samples were tested in triplicate.

\section{Evaluation of the Proteolytic Activity of Pancreatin in the Presence of Surfactants}

Stock solutions of Tween 80, Triton X-100, SDS and CTAB were prepared in Milli $Q$ water. The pancreatinsurfactant complexes containing the desired amount of surfactant (Table I) and with an initial proteolytic activity of 1750 USP units/L then were prepared. The activity of the pancreatin-surfactant preparations was assessed, as described in the "Assessment of the Proteolytic Activity of the Pancreatin Reagent" section, using appropriate controls. Experiments were conducted immediately after 
Table I. Surfactants and Their Concentrations Used for This Experiment

\begin{tabular}{lll}
\hline Type & Surfactant & Concentration $\%(w / v)$ \\
\hline Non-ionic & Polysorbate 80 (Tween 80) & 0.0005 \\
& & 0.0020 \\
& & 0.0500 \\
& & 0.5000 \\
& Polyethylene glycol tert- & 0.0050 \\
& octylphenyl ether & 0.0150 \\
& (Triton X-100) & 0.0500 \\
Anionic & Sodium dodecyl sulfate (SDS) & 0.2500 \\
& & 0.0500 \\
& & 0.2000 \\
Cationic & Cetyltrimethylammonium & 0.3000 \\
& bromide (CTAB) & 0.5000 \\
& & 0.0050 \\
& & 0.0500 \\
\hline
\end{tabular}

Concentration of the pancreatin was kept constant (1750 USP units of protease activity/L)

mixing the pancreatin and the surfactant, as well as after a 30 -min incubation time at $40^{\circ} \mathrm{C}$. All test samples were analyzed in triplicate.

For each series, a control experiment with a surfactantfree pancreatin solution was conducted to assess its proteolytic activity. This activity was considered to be $100 \%$.

The results are presented as percent relative activity along with \pm relative standard deviation, calculated from the three experiments for each tested surfactant at each concentration. The relative activity of pancreatin-surfactant complexes was calculated as a percentage of the activity of the surfactant-free pancreatin sample.

\section{RESULTS AND DISCUSSION}

All surfactants were tested in $50 \mathrm{mM}$ phosphate buffer solution at $\mathrm{pH} 7.5$ at four different concentrations: one below, one close to, and two above their corresponding $\mathrm{cmc}$ in water. The cmc value of each surfactant has been established in previously published experiments. The pancreatin concentration was kept constant, and its initial proteolytic activity (1750 USP units/L) corresponds to the suggested enzyme concentration in the two-tier USP procedure (13).

\section{Effect of Non-Ionic Surfactants on Pancreatin Proteolytic Activity}

As shown in Fig. 1a, the activity of pancreatin was preserved in the presence of Tween 80 even when the surfactant was added at a concentration 250-fold higher than its $\mathrm{cmc}(0.0020 \% \mathrm{w} / \mathrm{v})$ in water (20). Moreover, the catalytic efficiency of the proteolytic enzyme remained unaltered following a 30-min incubation of the pancreatin-surfactant mixture at $40^{\circ} \mathrm{C}$. It is likely that the non-ionic surfactant stabilizes the three-dimensional structure of the pancreatin proteases, preserves their correct folding, and therefore maintains their catalytic efficiency. Conversely, Tween 80 may also have a stabilizing effect on the substrate. In general, casein molecules having a highly unordered structure with a low content of $\alpha$-helical structural elements tend to aggregate (21). In a recent study, Ion Titapiccolo et al. have reported that two tested polysorbate emulsifiers (Tween 20 and Tween 80) modified the rheology of casein and have a direct effect on the size of casein micelles; however, the casein gelation point depends on the surfactant structure (22). Additionally, we assumed that Tween 80 was able to prevent the casein aggregation, therefore keeping the initial concentration of the substrate.

The cmc of Triton X-100 was reported to be $0.015 \%(w /$ v) (23). Dilute solutions of this surfactant $(0.01-0.1 \%(w / v))$ are often used in biotechnology during protein isolation to minimize protein-protein and protein-lipid interactions and to reduce the nonspecific bonding, therefore, to improve the final yield of the obtained proteins $(24,25)$. With Triton $\mathrm{X}-100$, we observed an increase in the pancreatin proteolytic activity in a concentration-dependent manner (Fig. 1b). Pancreatin activation was also observed for all pancreatinTriton X-100 complexes that were incubated at $40^{\circ} \mathrm{C}$ for $30 \mathrm{~min}$ prior to the test reaction. Our results are consistent with previously reported data reported for the effect of Triton
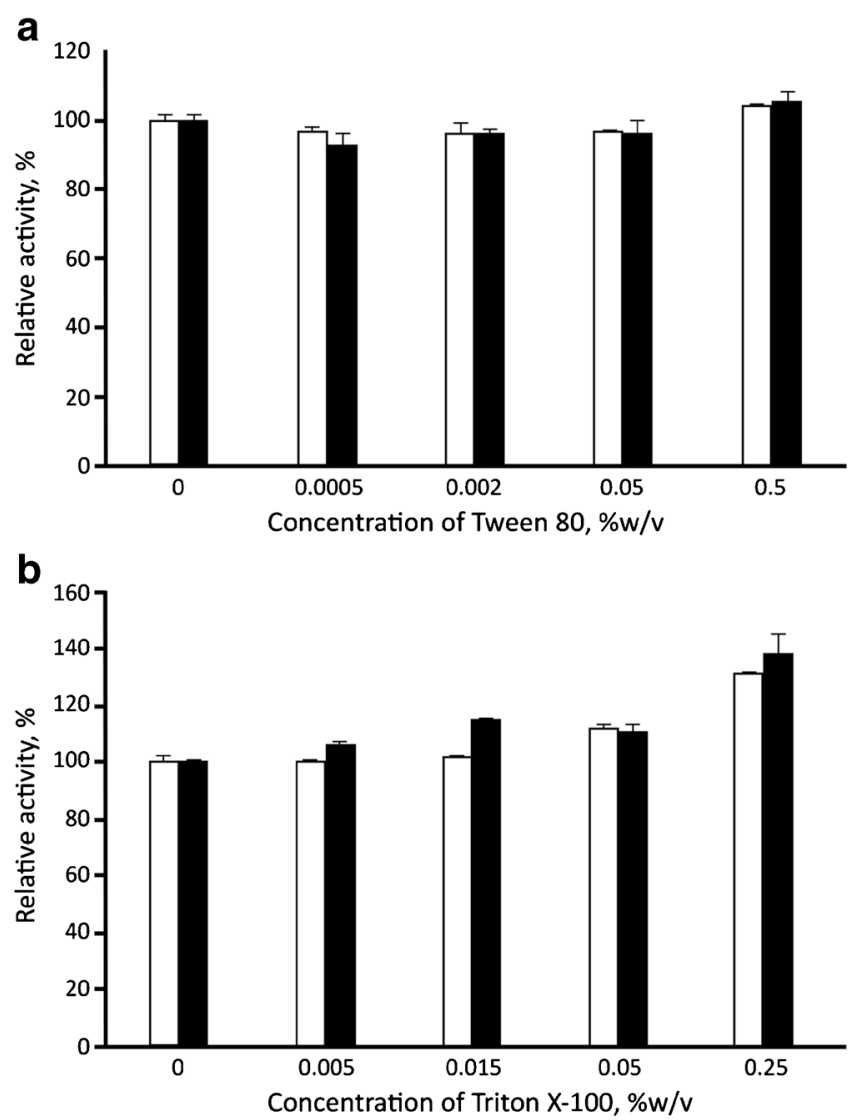

Fig. 1. Effect of tween-80 (a) and triton X-100 (b) on the activity of pancreatin. White rectangle represents assay test was carried out immediately after mixing pancreatin and the surfactant. Black rectangle represents assay test was carried out after incubation of the pancreatin-surfactant complexes at $40^{\circ} \mathrm{C}$ for $30 \mathrm{~min}$. The initial concentration of pancreatin is the same in each test reactions (1750 USP units of protease activity/L) 
X-100 on the catalytic activity of other proteolytic enzymes isolated from various sources (microbial, plant, and animal tissues). For example, carboxypeptidase from Penicillium janthinellum is increased by $35 \%$ in presence of Triton $\mathrm{X}-100$, and it was also shown that the surfactant had a cryoprotective effect for this enzyme (26).

On the other hand, Kubota et al. reported that high concentrations of Triton X-100 (1-10\%) did not effect, or had a negligible effect, on the activity of two carboxypeptidases isolated from the exocarp of mandarin oranges (27). At the same time, it has been shown that the activities of trypsin from Bacillus licheniformis and trypsin isolated from fish intestine (lane snapper) were inhibited by 20 and $14 \%$, respectively, in the presence of this surfactant $(28,29)$. Pancreatin, however, is a multicomponent enzyme mixture, and we cannot say what the influence of this surfactant on the individual enzymes is. It is likely that in the tested concentrations, Triton X-100 induced conformational changes near the binding sites of some of the proteinases that resulted in a more effective binding of the substrate molecules and subsequently led to an increase in the reaction rate of casein hydrolysis.

\section{Effect of Ionic Surfactants on Pancreatin Proteolytic Activity}

As expected, the two tested charged surfactants had stronger impact on pancreatin activity than the non-ionic surfactants, likely due to strong electrostatic interactions between the enzymes and surfactants.

We observed a rapid loss in the initial catalytic activity of pancreatin in the presence of SDS at a concentration fourfold lower than its cmc $(0.2 \% w / v)$ in water (Fig. 2a) (30). The relative proteolytic activity of pancreatin did not exceeded $50 \%$ at this lowest tested concentration of SDS, though further increase of the surfactant concentration resulted in a smoother decrease in the activity trend. This observed trend is in agreement with the previously document two- or multistep binding isotherms of SDS to some enzymes (16). We also observed a decrease in SDS solubility when it was added to the reaction media in a concentration greater than $0.5 \% \mathrm{w} / \mathrm{v}$; however, the reaction mixture became clear during the heating step of the experiment. Except for the carboxipeptidase, the other proteolytic enzymes that were present in the pancreatin have an isoelectric point (pI) higher than the $\mathrm{pH}$ of the reaction media; therefore, in the tested solution their surfaces are positively charged (31). This implies strong ionic interactions between the molecules of these enzymes and the SDS. Different changes in the volume and the shape of the protein-surfactant complexes occurred at different SDS concentrations, and the altered structures of the pancreatic enzymes can be directly linked to the observed change in their catalytic efficiency. The results are in agreement with the different models of structural organization of protein-surfactant complexes, as previously described (16).

Gosh reported that in the presence of trypsin, SDS aggregation occurs at a concentration approximately five times lower than the $\mathrm{cmc}$ of the pure surfactant in water (32). Gosh also observed protein unfolding and changes in the protein rheology in respect to the added amount of surfactant.
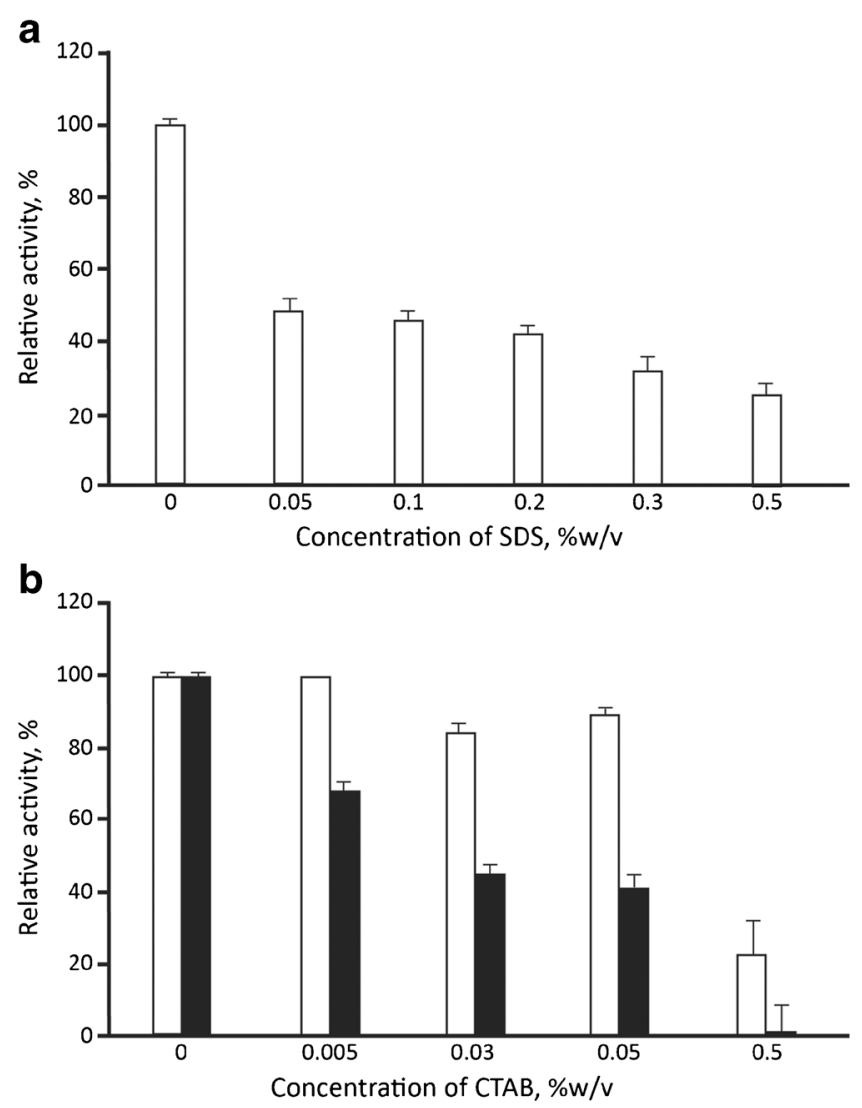

Fig. 2. Effect of SDS (a) and CTAB (b) on the activity of pancreatin. White rectangle represents assay test was carried out immediately after mixing pancreatin and the surfactant. Black rectangle represents assay test was carried out after incubation of the pancreatinsurfactant complexes at $40^{\circ} \mathrm{C}$ for $30 \mathrm{~min}$. The initial concentration of pancreatin is the same in each test reactions (1750 USP units of protease activity/L)

In our experiment, we did not detect any residual activity for the pancreatin-SDS complexes incubated at $40^{\circ} \mathrm{C}$ in each of the tested SDS concentrations. It appears that the thermal treatment of the samples increased the unfolding rate of the proteinases that comprise the pancreatin. In comparison, Prasad et al. reported that a 30-min incubation of trypsin at room temperature with various amounts of SDS (trypsin/SDS ratio from $1: 10$ to $1: 80$ ) reduced its initial activity by $61 \%$ (33).

In addition, we assume that the casein substrate may play a protective role in the enzymes against alteration by surfactant. Porter and Peterson also observed this phenomenon, as the SDS-induced trypsin and chymotrypsin denaturation is slowed in presence of macromolecular substrates (34). There are also data in the literature that suggests that the addition of a sucrose, trehalose, or trypsin inhibitor to trypsin solutions may minimize the destabilizing effect of SDS and reduced the trypsin autoproteolysis $(33,35)$.

The effect of [CTAB]/[pancreatin] ratio on the pancreatin proteolytic activity is shown in Fig. 2b. In contrast to SDS, adding CTAB to the pancreatin solution in a concentration below its $\mathrm{cmc}(0.030 \% w / v)$ did not affect the pancreatin activity (30). A moderate decrease in pancreatin efficiency was observed for samples with the CTAB concentration near the $\mathrm{cmc}$; however, further increases of the 
amount of surfactant significantly inhibited the proteolytic activity of pancreatin. The residual activity of the samples that contain $0.25 \%$ of CTAB did not exceeded $40 \%$ of the control sample without surfactant. We also observed a formation of precipitates when the amount of cationic surfactant exceeded $0.375 \%$, which is probably due to lowering of the solubility of the proteins (enzymes and/or the substrate) that are present in the reaction mixture. A similar but more pronounced effect was observed for the thermally incubated pancreatin-CTAB samples.

Celej et al. found that conformational changes occurred in the molecule of $\alpha$-chymotrypsin in the presence of CTAB micelles and resulted in enzyme superactivation when tested at $25^{\circ} \mathrm{C}$ using a chromogenic model substrate, though the monomeric CTAB had no effect on the enzyme activity (36). Incubation of $\alpha$-chymotrypsin and CTAB at room temperature for $2 \mathrm{~h}$, however, resulted in a decrease in the hydrolytic activity of the samples, in comparison to the reaction conducted with surfactant-free enzyme. This alteration was ascribed to an increase of the rate of selfproteolysis, a by-reaction for trypsin and chymotrypsin (34). Spreti et al. also observed a change in the kinetic parameters of $\alpha$-chymotrypsin-catalyzed hydrolysis of model substrates that they explained was due to CTABinduced conformational changes in the enzyme molecule (37). Spreti et al. also found that in the presence of CTAB, two different substrates may have different substrateassociation constant, i.e., different substrate distributions between the aqueous phase and the micelles that suggested differences in their effective concentration during the chymotrypsin-catalyzed hydrolysis, and is one of the reasons for the estimated 36-fold difference in the specificity constants. In a recent paper, a two- to sixfold increase in the hydrolytic activity of trypsin in the presence of three cationic surfactants, including CTAB, with respect to their concentration and the structure of the substrates was reported (38). It is noteworthy to mention that there are no comprehensive studies on the effect of ionic surfactants on elastases and carboxypeptidase, the other two proteolytic enzymes that are present in pancreatin.

Conversely, the substrate casein may also interact with the surfactants that are present in the solution. Casein is the group name for the most abundant class of milk proteins. They are characterized by a highly random structure and $\mathrm{pI}$ in the range between 4.2 and 5.1 (21). Using far ultraviolet circular dichroism and fluorescent spectroscopy, Chakaborty and Basak showed that in the presence of CTAB and SDS, casein folding is promoted and the protein molecules adopted a more ordered conformation (39). At pH 7.5, the casein is negatively charged and interacts effectively with CTAB that resulted in increase of the content of $\alpha$-helical secondary structural elements and "necklace and bead" proteinsurfactant micelle-like structures. The nature of the interactions between the negatively charged casein molecules and the anionic surfactant, however, are different. SDS micelles nucleate around the non-polar/hydrophobic regions of the substrate and are "rod-like"; other ordered structures are also observed (39). We assumed that for these two more ordered and/or folded structures; however, the scissile bonds become hindered and in some cases inaccessible to the catalytic centers of the proteolytic enzymes, which can also affect the catalysis of the casein molecules. It is likely that along with the denaturation of the enzymes in the pancreatin complex, a folding or re-organization in casein molecules occur in presence of ionic surfactants that simultaneously caused the dramatic reduction in the pancreatin proteolytic activity.

\section{CONCLUSION}

Pancreatin exhibits remarkable stability in the presence of the either tested non-ionic surfactant even after prolonged heating $\left(30 \mathrm{~min}, 40^{\circ} \mathrm{C}\right)$. Tween- 80 and small amounts of Triton X-100 preserve its proteolytic activity, and the latter added at concentrations above $\mathrm{cmc}$ also has a stimulatory effect on the pancreatin activity. In contrast, the two tested ionic surfactants deteriorate the catalytic efficiency of the pancreatin at the tested conditions. The effect depends on the concentration of the surfactants and the incubation time. SDS quickly inactivates the pancreatin even at concentrations lower than its $\mathrm{cmc}$ in water. CTAB, at concentrations below or near the $\mathrm{cmc}$, has either no or a slightly inhibitory effect on casein hydrolysis when tested immediately after mixing with pancreatin.

Based on these observations, we believe that in the presence of non-ionic surfactants, the dissolution testing of highly cross-linked gelatin capsules can be conducted following a one-step procedure. However, when ionic surfactants are being used in the dissolution medium, they should not be mixed simultaneously with the pancreatin, and in these cases an enzyme pre-treatment phase should be considered.

\section{ACKNOWLEDGMENTS}

M. G. would like to acknowledge USP for the visiting scientist fellowship.

Open Access This article is distributed under the terms of the Creative Commons Attribution 4.0 International License (http://creativecommons.org/licenses/by/4.0/), which permits unrestricted use, distribution, and reproduction in any medium, provided you give appropriate credit to the original author(s) and the source, provide a link to the Creative Commons license, and indicate if changes were made.

\section{REFERENCES}

1. Elzoghby AO. Gelatin-based nanoparticles as drug and gene delivery systems: reviewing three decades of research. J Control Release. 2013;172(3):1075-91.

2. Lee SJ, Yhee JY, Kim SH, Kwon IC, Kim K. Biocompatible gelatin nanoparticles for tumor-targeted delivery of polymerized siRNA in tumor-bearing mice. J Control Release. 2013;172(1):358-66.

3. Cole G. Evaluating development and production costs: tablets versus capsules. Pharm Technol Eur. 1998;5:17-26.

4. Schrieber R, Gareis H. From collagen to gelatine In: Gelatine handbook: theory and industrial practice, Weinheim, Germany:Wiley-VCH; 2007. p. 45-117.

5. Changez M, Burugapalli K, Koul V, Choudhary V. The effect of composition of poly(acrylic acid)-gelatin hydrogel on gentamicin sulphate release: in vitro. Biomaterials. 2003;24(4):527-36. 
6. Ding D, Jiang X. Drug delivery from protein-based nanoparticles In: Gu S, editor. Bioinspired and biomimetic polymer systems for drug and gene delivery. Weinheim, Germany: Chemical Industry Press and Wiley-VCH; 2015. p. 149-70.

7. Gray VA, Cole E, Riva Toma JMD, Ghidorsi L, Guo J-H, Han J$\mathrm{H}$, et al. Use of enzymes in the dissolution testing of gelatin capsules and gelatin-coated tablets-revisions to dissolution $<711>$ and disintegration and dissolution of dietary supplements $<2040>$. Dissolut Technol;2014:6-19.

8. Singh S, Rama Rao KV, Venugopal K, Manikandan R. Alteration in dissolution characteristics of gelatin-containing formulations. A review of the problem, test methods, and solutions. Pharm Tech. 2002;36-58.

9. Marques MRC. Enzymes in the dissolution testing of gelatin capsules. AAPS Pharm Sci Technol. 2014;15(6):1410-16.

10. Meyer MC, Straughn AB, Mhatre RM, Hussain A, Shah VP, Bottom CB, et al. The effect of gelatin cross-linking on the bioequivalence of hard and soft gelatin acetaminophen capsules. Pharm Res. 2000;17(8):962-6.

11. Cole ET, Cadé D, Benameur H. Challenges and opportunities in the encapsulation of liquid and semi-solid formulations into capsules for oral administration. Adv Drug Deliv Rev. 2008;60(6):747-56.

12. $<711>$ Dissolution. In: The United States Pharmacopeia and National Formulary, USP38-NF33. Rockville, MD; The United States Pharmacopeial Convention: 2015. p. 487.

13. <2040> Disintegration and dissolution of dietary supplements. In: The United States Pharmacopeia and National Formulary, USP38--NF33. Rockville, MD; The United States Pharmacopeial Convention: 2015. p. 1778.

14. <1094> Capsules - dissolution testing and related quality attributes. In: The United States Pharmacopeia and National Formulary, USP38-NF33. Rockville, MD; The United States Pharmacopeial Convention: 2015. p 1101.

15. Zhao F, Malayev V, Rao V, Hussain M. Effect of sodium lauryl sulfate in dissolution media on dissolution of hard gelatin capsule shells. Pharm Res. 2004;21(1):144-8.

16. Otzen D. Protein-surfactant interactions: a tale of many states. Biochim Biophys Acta. 2011;1814(5):562-91.

17. Guzman ML, Marques MR, Olivera ME, Stippler ES. Enzymatic activity in the presence of surfactants commonly used in dissolution media, part 1: pepsin. Results Pharma Sci. 2016;6:15-9.

18. Berdutina AV, Neklyudov AD, Ivankin AI, Karpo BS, Mitaleva SI. Comparison of proteolytic activities of the enzyme complex from mammalian pancreas and pancreatin. Appl Biochem Microbiol. 2000;36(4):363-7.

19. Pancreatin In: In: The United States Pharmacopeia and National Formulary, USP38-NF 3, Second Supplement, Rockville, MD; United States Pharmacopeial Convention: 2016. p. 4740-42.

20. Steele RH, Limaye S, Cleland B, Chow J, Suranyi MG. Hypersensitivity reactions to the polysorbate contained in recombinant erythropoietin and darbepoietin. Nephrology (Carlton). 2005;10(3):317-20.

21. Modler HW. Functional properties of nonfat dairy ingredients-a review. Modification of products containing casein. J Dairy Sci. 1985;68(9):2195-205.

22. Ion Titapiccolo G, Corredig M, Alexander M. Modification to the renneting functionality of casein micelles caused by nonionic surfactants. J Dairy Sci. 2010;93(2):506-14.

23. Tiller GE, Mueller TJ, Dockter ME, Struve WG. Hydrogenation of Triton X-100 eliminates its fluorescence and ultraviolet light absorption while preserving its detergent properties. Anal Biochem. 1984;141(1):262-6.

24. Kalipatnapu S, Chattopadhyay A. Membrane protein solubilization: recent advances and challenges in solubilization of seroto$\operatorname{nin}_{1 \mathrm{~A}}$ receptors. IUBMB Life. 2008;57(7):505-51.

25. Tao H, Liu W, Simmons B, Harris H, Cox T, Massiah M. Purifying natively folded proteins from inclusion bodies using sarkosyl, Triton X-100, and CHAPS. Biotechniques. 2010;48(1):61-4.

26. Yokoyama S, Miyabe T, Oobayashi A, Tanabe O, Ichishima E. Stabilization of crystalline acid carboxypeptidase from Penicillium janthinellum by nonionic surfactants, and inhibition of enzyme activity by anionic compounds. Agric Biol Chem. 1977;41(8):1379-83.

27. Kubota Y, Funakoshi T, Shoji S, Moriyama M, Ueki H. Carboxypeptidases from the exocarp of mandarin orange (Citrus unshiu MARC.). II. Chemical and enzymatic properties of carboxypeptidases Cua and Cub. Chem Pharm Bull. 1979;28(12):3479-87.

28. Gong J-S, Li W, Zhang D-D, Xie M-F, Yang B, Zhang R-X, et al. Biochemical characterization of an arginine-specific alkaline trypsin from Bacillus licheniformis. Int $\mathrm{J}$ Mol Sci. 2015;16(12):30061-74.

29. Espósito T, Marcuschi M, Amaral I, Carvalho Jr LB, Bezerra RS. Trypsin from the processing waste of the lane snapper (Lutjanus synagris) and its compatibility with oxidants, surfactants and commercial detergents. J Agric Food Chem. 2010;58(10):6433-9.

30. Aguiar J, Carpena P, Molina-Bolívar JA, Carnero RC. On the determination of the critical micelle concentration by the pyrene $1: 3$ ratio method. J Colloid Interface Sci. 2003;258:116-22.

31. Rao MB, Tanksale AM, Ghatge MS, Deshpande VV. Molecular and biotechnological aspects of microbial proteases. Microbiol Mol Biol Rev. 1998;62(3):597-635.

32. Ghosh S. Interaction of trypsin with sodium dodecyl sulfate in aqueous medium: a conformational view. Colloid Surf B Biointerfaces. 2008;66(2):178-86.

33. Prasad S, Roy I. Effect of disaccharides on the stabilization of bovine trypsin against detergent and autolysis. Biotechnol Prog. 2010;26(3):627-35.

34. Porter WH, Preston JL. Retention of trypsin and chymotrypsin proteolytic activity in sodium dodecyl sulfate solutions. Anal Biochem. 1975;66(1):69-77.

35. Sweadner KJ. Trypsin inhibitor paradoxically stabilizes trypsin activity in sodium dodecyl sulfate, facilitating proteolytic fingerprinting. Anal Biochem. 1991;194(1):130-5.

36. Celej MS, D'Andrea MG, Campana PT, Fidelio GD, Bianconi ML. Superactivity and conformational changes on alphachymotrypsin upon interfacial binding to cationic micelles. Biochem J. 2004;378(Pt 3):1059-66.

37. Spreti N, Alfani F, Cantarella M, D'Amico F, Germani R, Savelli G. $\alpha$-Chymotrypsin superactivity in aqueous solutions of cationic surfactants. J Mol Catal B Enzymol. 1999;6(1-2):99-110.

38. Verma SK, Ghosh KK, Verma R, Xiang W, Li N, Zhao X. Surface, conformational and catalytic activity approach of $\alpha$ chymotrypsin and trypsin in micellar media. Colloids Surf A Physiochem Eng Asp. 2015;470:188-93.

39. Chakraborty A, Basak S. Effect of surfactants on casein structure: a spectroscopic study. Colloid Surf B Biointerfaces. 2008;63(1):83-90. 